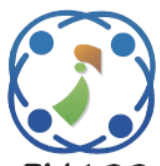

\title{
Optimization of Task Scheduling Algorithm Using Modified Mean Grey-Wolf
}

\author{
Md. Yusuf Mulge ${ }^{1 *}$ \\ ${ }^{1}$ CVR College of Engineering, Telengana, India \\ * Corresponding author's Email: dryusufmulge@ gmail.com
}

\begin{abstract}
Task Scheduling (TS) is used to improve the performance of Cloud Computing (CC) and optimizing the Quality of Service (QoS) of the cloud. The main objective of this research work is to optimize the TS by minimizing the MakeSpan (MS) and energy consumption. The scheduling issues are overcome by Modified Mean Grey Wolf Optimization algorithm (MGWO) and it enhances the overall performance of the system. In this MGWO, the leadership hierarchy is simulated by four grey wolves such as alpha, beta, delta and omega for the major steps like hunting, searching, encircling and attacking the prey. Here, hunting and encircling modified using the mean value to improve the effectiveness of GWO. CloudSim toolkit is used for evaluating the objective of the Modified-MGWO in standard workload (left-skewed \& right-skewed dataset). The proposed Modified-MGWO algorithm reduced energy consumption nearly $10 \%$ when compared with the existing algorithms such as Particle Swarm Optimization (PSO) and standard GWO. Moreover, the MS of the Modified-MGWO method reduced nearly $13 \%$ when compared with existing methods for both datasets.
\end{abstract}

Keywords: Cloud computing, CloudSim, Grey wolf optimization, Makespan, Quality of service, Task scheduling.

\section{Introduction}

In recent years, $\mathrm{CC}$ becomes more popular among the communities of business and research areas because of its virtualization technologies [1]. According to on-demand resources, cloud provides a service of optimally scheduling resources and virtualize those for assigning tasks [2]. The main challenge in the heterogeneous cloud environment is scheduling the workload because of limited cloud resources with changing functionalities and capacities. The profit of Cloud Service Providers (CSP) should be increased by allocating user tasks while guaranteeing QoS for all tasks which is considered as a key issue in scheduling the task $[3,4]$. The cloud resources such as TS should meet the user requirements for maximizing the economic benefits of CSP [5, 6]. As a service business model, the TS meets the QoS requirements of user tasks, it includes cost, MS and deadline in clouds [7]. The overall performance of clouds can be degraded by improper matching applications on some hardware platforms such as Application Programming Interfaces (APIs),
IBM hardware, etc. [8, 9]. Several classical scheduling algorithms used for scheduling task on resources, such as Min-Min [10], Max-Min [11], and first-in-first-out (FIFO). These classical algorithms aren't feasible because it initially requires to build all possible task schedulers and compare them with one another to find the best solution which would take more time [12-14]. Some researchers used intelligent optimization algorithms to find an approximate optimal solution for tasks. These algorithms can reduce the search space and ensure the execution within a feasible operational time, which provides a balance between their running time and resultant schedule's optimality. Optimal resource scheduling considers as a basic influence of CC [15], which can be achieved by hybridization of greedy approaches with meta-heuristic algorithms. Particle swarm optimization [16], genetic algorithms, ant colony and Cuckoo search [17] are the examples of metaheuristic algorithms. In this paper, a cloud TS algorithm based on Modified meta heuristic Modified-MGWO is proposed for reducing the consumption of energy and execution time of the task 
in the cloud. The CloudSim tool is used to evaluate the performance of MGWO for some common workload in simulated data centres. The main aims of this paper are as follows:

- Formulate the MS through mathematical models for optimal TS and reduce the consumption of energy.

- Develop the Modified-MGWO for optimizing TS in Infrastructure as a service (IaaS) CC.

- Implement the proposed Modified-MGWO algorithm in CloudSim simulation tool.

- Performance evaluation of the existing metaheuristic algorithms with Modified-MGWO optimization algorithm obtained by considering the metrics of MS, and energy consumption $(\%)$.

- Comparative and statistical analysis of the Modified-MGWO algorithm with existing meta-heuristic algorithms for TS in IaaS CC.

The rest of the paper is organized as follows: The related works for TS algorithms with its limitations are discussed in section 2. The framework of TS is discussed in section 3. Section 4, provides the description of the proposed Modified MGWO algorithm. Section 5 presents the results and discussion of the proposed method with the help of experimental analysis. The conclusion of this paper is made in section 6 .

\section{Literature review}

Researchers developed numerous methodologies on TS. In this sub-section, a brief evaluation of a few essential contributions to the existing literatures is presented.

H. Yuan, J. Bi, W. Tan, M. Zhou, B. H. Li, and J. Li [18] dispatched all receiver tasks to public and private Cloud Data Centre (CDC) by implementing a Temporal TS algorithm (TTSA). The hybrid simulated-annealing PSO solved the problem of mixed integer linear program which is also known as cost minimization problem in every iteration of TTSA. While meeting the delay bounds of tasks, the cost of private CDC was reduced and throughput was increased by TTSA. But, the important characteristics of hybrid clouds such as dispatching time and execution delay were not described by this approach.

H. B. Alla, et al., [19] proposed an approach based on hybrid meta-heuristic algorithms and Dynamic Dispatch Queues (TSDQ) for TS optimization. Fuzzy Logic with PSO algorithm
(TSDQ-FLPSO) and Simulated Annealing with PSO algorithm (TSDQ-SAPSO) was proposed by this approach for optimizing the task in an effective way. The experiments were carried out in an open source simulator CloudSim on real data sets and synthetic data from real systems. The optimal result of this approach provided better results, especially in a high dimensional problem. Both TSDQ-FLPSO and TSDQ-SAPSO algorithms requires maximum task completion for optimization.

J.A.J. Sujana, T. Revathi, T.S. Priya, and K. Muneeswaran [20] focused to minimize the MS and scheduling the task effectively with the help of Smart PSO (SPSO). A Variable Neighbourhood PSO overcame the local optima problem, and experiments were carried-out in CloudSim simulator. With a minimum number of iterations, the SPSO method yielded better MS than the existing methods with assured security. The SPSO algorithm gradually increases the energy cost in a heterogeneous environment.

C. Thirumalaiselvan, and V. Venkatachalam, [21] implemented a Rate Based Scheduling (RBS) algorithm in multi-cloud environment for TS. The tasks were rated by RBS algorithm and maximum rated tasks were allocated to the Virtual Machine (VM). If the stack was full, then the cloud manager checked the priority of the task. The energy consumption cost and delay were reduced by RBS algorithm. The major limitation of RBS algorithm was the MS gradually increased in the multi-cloud environment.

G. Natesan, and Arun Chokkalingam, [22] proposed an oppositional grey wolf's optimization (OGWO) scheduling method based on cost and time model on CC. The computational speed and convergence profile were enhanced by opposition based learning with standard GWO. The experimental results stated that the OGWO provided better quality schedules with less computation time and memory utilization. But, OGWO approach takes more time for mapping the task to physical machines.

\section{Framework of task scheduling algorithm}

TS is one of the important aspects in the CC resource management of designing and providing scheduling framework in IaaS clouds, which is explained in this section. The main aim of the CC is to improve the load balancing as well as QoS to provide the entire cloud system and users with optimal operation time. Finally, the aim of CC is to provide optimal scheduling of the tasks on all resources. The optimal matching tasks and resources 


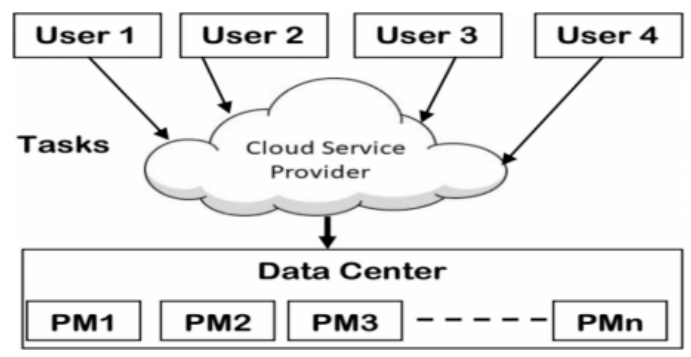

Figure.1 Cloud architecture

are provided by TS which are closely related to load balancing in cloud environment [23]. The minimization of energy and MS is obtained by scheduling a suitable task on resources in the cloud environment. Fig. 1 depicts the entire TS framework consists of four main components, namely task scheduler, user portal, cloud data centre with physical machines (PM) and information service.

The unit of task is submitted by the user interface which is provided by user portal. This task can be executed in PM by further dividing the units into small tasks. The scheduler schedules the task to PM in the data centre by having the detailed information about resource utilization and other log information which is provided by information service. The task units are accepted by the scheduler from the user portal and select the appropriate PM by using Information Service in the data centre. Once the execution is completed, the new status of resource and its results will be sent back to service for another scheduling. The task is allocated to data centres by using proposed Modified-MGWO optimization algorithm to improve the efficiency of the system.

\section{Proposed methodology}

In this paper, a modified MGWO algorithm is proposed which is used for increasing the accuracy and performance of the GWO algorithm. In Modified-MGWO method, where the encircling and hunting operations have been modified by adding mean value $\theta$ to the equations. The remaining equations/procedures are similar to standard GWO algorithm [24]. The MGWO algorithm mainly concentrated in two areas which are described below, it is used for improving the efficiency of each wolf's motion and suitable path in searching area.

\subsection{Encircling prey}

The grey wolves encircled the prey during hunting and that can be improvised by using the following Eqs. (1) and (2).

$$
\vec{D}=\left|\vec{C} \cdot \overrightarrow{X_{p}}(t)-\theta \cdot(\vec{X}(t))\right|
$$

$$
\vec{X}(t+1)=\overrightarrow{X_{p}}(t)-\vec{A} \cdot \vec{D}
$$

Where, $\theta$ is the mean, $\overrightarrow{X_{p}}$ denotes the position vector of prey, $t$ is the present iteration, $\vec{X}(t)$ represents the position vector of grey wolf. The vector $\vec{A}$ and $\vec{C}$ are denotes as Eq. (3) \& (4).

$$
\begin{aligned}
& \vec{A}=2 \vec{a} \cdot \overrightarrow{r_{1}}-\vec{a} \\
& \vec{C}=2 \cdot \overrightarrow{r_{2}}
\end{aligned}
$$

Where element of $\vec{a}$ decreases from 2 to $0 . \overrightarrow{r_{1}}, \overrightarrow{r_{2}}$ takes certain random values lying between $[0,1]$.

\subsection{Hunting}

The groups such as $\alpha, \beta$ and $\delta$ are irregularly participated and guide for hunting the prey. The optimal and best candidate can be represented as $\alpha, \beta, \delta$ and the remaining solutions are denoted by $\omega$. The positions of mean are calculated in search space region to improve each wolfs position. The Eq. 5-11 will explain below.

$$
\begin{aligned}
& \overrightarrow{D_{\alpha}}=\left|\overrightarrow{C_{1}} \cdot \overrightarrow{X_{\alpha}}-\theta \cdot \vec{X}(t)\right| \\
& \overrightarrow{D_{\beta}}=\left|\overrightarrow{C_{2}} \cdot \overrightarrow{X_{\beta}}-\theta \cdot \vec{X}(t)\right| \\
& \overrightarrow{D_{\delta}}=\left|\overrightarrow{C_{3}} \cdot \overrightarrow{X_{\delta}}-\theta \cdot \vec{X}(t)\right| \\
& \overrightarrow{X_{1}}=\overrightarrow{X_{\alpha}}-\overrightarrow{A_{1}} \cdot\left(\overrightarrow{D_{\alpha}}\right) \\
& \overrightarrow{X_{2}}=\overrightarrow{X_{\beta}}-\overrightarrow{A_{2}} \cdot\left(\overrightarrow{D_{\beta}}\right) \\
& \overrightarrow{X_{3}}=\overrightarrow{X_{\delta}}-\overrightarrow{A_{3}} \cdot\left(\overrightarrow{D_{\delta}}\right) \\
& \vec{X}(t+1)=\frac{\overrightarrow{X_{1}}+\overrightarrow{X_{2}}+\overrightarrow{X_{3}}}{3}
\end{aligned}
$$

\subsection{Fitness function}

The execution time and consumption of energy can be optimized by scheduling tasks in the cloud environment. The quality of the solutions is evaluated by calculating the fitness function to achieve the objectives of this research work. But, the nearest optimal solution must find out by fitness function for every optimization problem. The objective functions of each search agents are used to calculate the fitness function. The fitness function for energy and time constraints is given in Eq. (12).

$$
F F(I)=\alpha \times F_{1}^{\text {min }}+(1-\alpha) \times F_{2}^{\text {min }}
$$


Where, $F F(I)$ stands for fitness function. Here $\alpha$ is a constant value, the value of $\alpha$ is between $1>$ $\alpha \geq 0$. The execution time can be minimized by first task objective which is denoted as $F_{1}^{\mathrm{min}}$. The consumption of energy can be further reduced by the second objective which is represented as $F_{2}^{\min }$.

\subsection{Pseudo code of modified MGWO}

\section{Input:}

Parameters of Modified MGWO Algorithm. Task $\left(T_{y}\right) \quad$ and Resource $\quad\left(R_{x}\right) \forall x \in$
$\{1,2, \ldots m\}$ and $y \in\{1,2, \ldots \ldots, n\}$.

\section{Output:}

The tasks are scheduled to an optimal resource $\left\{T_{y}, R_{x}\right\}$.

\section{Parameters initialization:}

1. ' $\mathrm{n}$ ' is an original population size $\overrightarrow{X_{l}}=(i=$ $1,2, \ldots, n)$, factor "a", C \& A coefficient vector, $\operatorname{Max}_{i t}$ rmaximum iteration.

2. Set counter preliminary value $t=0$

\section{Population initialization:}

3. Set $i=1$

4. While $(i \leq n)$ do

5. Randomly generate initial population $\overrightarrow{X_{l}(t)}$

6 . Compute the fitness of every single grey wolf in the search area using Eq. (12)

\section{End While}

8. The grey wolf with most fitness is denoted as Alpha $\overrightarrow{X_{\alpha}}$.

9. The grey wolf with the second most fitness is denoted as Beta $\overrightarrow{X_{\beta}}$.

10. The grey wolf with the third most fitness is denoted as Delta $\overrightarrow{X_{\delta}}$.

\section{Solution Updating phase}

11. While $(t<$ Maxitr $)$

of iterations

//Maximum number

12. For each search agent

13. Using Eq. (11) each grey wolf position is updated.

14. End for

15. The value of "a" is decrease for 2 to 0 .

16. Using Eq. (1) and (2) the coefficient of "A" and " $\mathrm{C}$ " are updated respectively.

17. Compute the fitness value of all grey wolfs using Eq. (12).

18. Update the positions of Alpha, Beta and Delta using Eqs. (8), (9) and (10).

19. set $t=t+1 \quad / /$ increasing the iteration counter 20. End while

\section{Best Solution}

21. return Alpha $\overrightarrow{X_{\alpha}}$ as the nearest optimal solution from the search space

\subsection{Implementation steps}

Step 1: Define the related parameter values in the algorithm and randomly generate the initial population.

Step 2: Code the particles and initialize the population.

Step 3: Randomly divide the group into two subgroups, and adopt different processing methods for different subgroups.

Step 4: For each subgroup, specific algorithm is utilized to update the position and speed of the particles. After that, the two subgroups are integrated. Step 5: Judge whether iterative conditions can meet the stop conditions or achieve the iteration times.

Step 6: If iterative conditions cannot meet the stop conditions or achieve the iteration times, execute step 3. Otherwise, execute step 7.

Step 7: Select the top $10 \%$ of individuals with the best fitness values from Step 4 and generate the initial pheromone of the grey wolf algorithm.

Step 8: Establish the TS model of the grey wolf algorithm and initialize the parameters of the algorithm.

Step 9: Every grey wolf selects transfer nodes, updates the local pheromone and adds the nodes selected to the tabu list.

Step 10: When all the grey wolf completes a cycle, the global pheromone should be updated.

Step 11: Evaluate whether iteration conditions can meet the stop conditions or achieve the iteration times.

Step 12: If the iterative conditions cannot meet the stop conditions or achieve the iteration times, it will execute step 11. Otherwise, the global best solution is obtained.

\section{Experimental outcomes}

This section explains the computational experiments for evaluating the performance of Modified-MGWO with existing methods such as PSO and standard GWO. CloudSim toolkit based on JAVA is used for simulation with the configuration of computer with 8GB RAM, 64-bit Windows OS, Intel(R) Core(TM) i5-457 and 4 CPU @ 2.9 GHz.

\subsection{Measures of effectiveness}

In the process of $\mathrm{TS}$, various parameters considered as performance metrics such as MS, cost, flow time, waiting time, tardiness, turnaround time, 
throughput, load balancing, resource utilization, etc. Optimized performance metrics are defined as follows.

\subsubsection{Makespan}

It Indicates the time taken for executing all tasks (i.e.: the finishing time of the last task). This metric can be calculated by Eq. (13).

$$
\text { Makespan }=\max _{i \in \operatorname{tasks}}\left\{F T_{i}\right\}
$$

Where $F T_{i}$ denotes the finishing time of task $i$.

\subsubsection{Energy consumption}

The number of data centres is directly proportional to the energy consumption which is also considered as one of the objectives of this paper. As the number of data centres decreases, then the energy factor also decreases. The consumption of energy can be evaluated by multiplying the matrix element by VM consumed energy is defined by Eq. (14).

$$
E_{c}=\frac{1}{V M \times X} \sum_{i=1}^{m} \sum_{j=1}^{n} r_{i j} \times d_{i j}
$$

Where $r_{i j}$ represents a matrix element, at the time of execution, energy utilized by every resource can be denoted as $d_{i j}$ and number of tasks can be represented as $X$. The objective of energy consumption function is represented by Eq. (15).

$$
F^{\min }=E_{c}
$$

\subsection{Dataset description}

In this research, two different datasets used in order to evaluate the performance of ModifiedMGWO technique. Two types of datasets such as Left Skewed and Right Skewed (LS-RS) used for validating the effectiveness of Modified-MGWO, in that each dataset has 100-500 tasks. The RS dataset contains more small sized tasks and few large sized tasks, whereas LS contains few data in both small and large sized tasks.

\subsection{Evaluation of Makespan}

The MS parameter is used to check the performance result of the proposed modified-MGWO. The proposed work is compared with PSO [20] and standard GWO [22] for TS. The tasks executed nearly 30 times and $50 \mathrm{VMs}$ used in the simulation part for all LS-RS dataset. Table 1 represents the average MS of executing tasks by using ModifiedMGWO, PSO and standard GWO. The graphical representation of the proposed method for both LS and RS datasets are described in Fig. 2 and 3.

For LS, when the number of cloud tasks is 200, the MS value for modified MGWO, PSO and standard GWO are 130, 145 and 150. Similarly, when the number of cloud tasks is 400 , the MS values for modified MGWO, PSO and standard GWO are 280, 325 and 341. The MS values of proposed Modified MGWO, PSO and standard GWO are 394, 463 and 340 when the number of cloud is 500. The values of MS are high for 500 tasks and low for 100 tasks. The experimental results stated that the existing method PSO achieved 73 MS, standard GWO achieved $64 \mathrm{MS}$ and proposed MGWO achieved 60 MS for the cloud task 100.

Similarly, for the RS dataset, when the number of cloud tasks is 100 , the MS values are 52, 78 and 70 for Modified MGWO, PSO and GWO. However, when the number of cloud tasks is 300 , the MS values are 185, 210 and 198 for proposed method, PSO and GWO respectively. The existing method PSO achieved 115 MS, standard GWO achieved 107 MS and proposed MGWO achieved 105 MS for cloud task 200. In RS, the MS improvements on PSO and standard GWO are given as 10.16 and 4.664 respectively. The improvements of MS values produced by the proposed Modified MGWO are better when compared with PSO and standard GWO.

\subsection{Evaluation of energy consumption}

Table 2 describes the energy consumption of proposed method for both LS-RS datasets. Fig. 3 and 4 depicts an energy consumption of LS-RS dataset using Modified MGWO, PSO and standard GWO.

Table 1. Performance evaluation for Makespan of proposed method

\begin{tabular}{|c|c|c|c|c|c|c|}
\hline \multirow{2}{*}{$\begin{array}{c}\text { Number of } \\
\text { tasks }\end{array}$} & \multicolumn{2}{|c|}{ PSO } & \multicolumn{2}{c|}{ Standard GWO [22] } & \multicolumn{2}{c|}{ Modified MGWO } \\
\cline { 2 - 7 } & Left skewed & Right skewed & Left skewed & Right skewed & Left skewed & $\begin{array}{c}\text { Right } \\
\text { skewed }\end{array}$ \\
\hline 100 & 73 & 78 & 64 & 70 & 60 & 52 \\
\hline 200 & 145 & 115 & 150 & 107 & 130 & 105 \\
\hline 300 & 210 & 192 & 198 & 200 & 190 & 185 \\
\hline 400 & 325 & 260 & 341 & 250 & 280 & 240 \\
\hline 500 & 463 & 340 & 424 & 328 & 394 & 310 \\
\hline
\end{tabular}




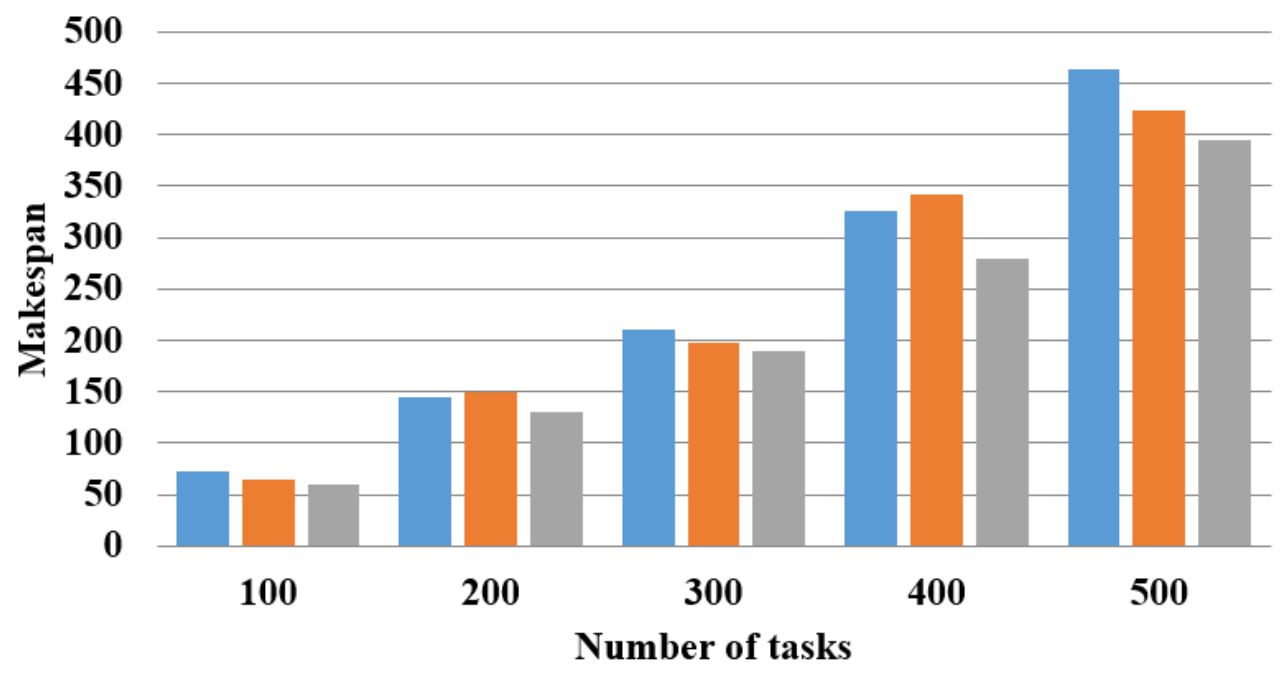

$\square$ PSO $\backsim$ Standard GWO Modified MGWO

Figure.2 Makespan for left skewed dataset

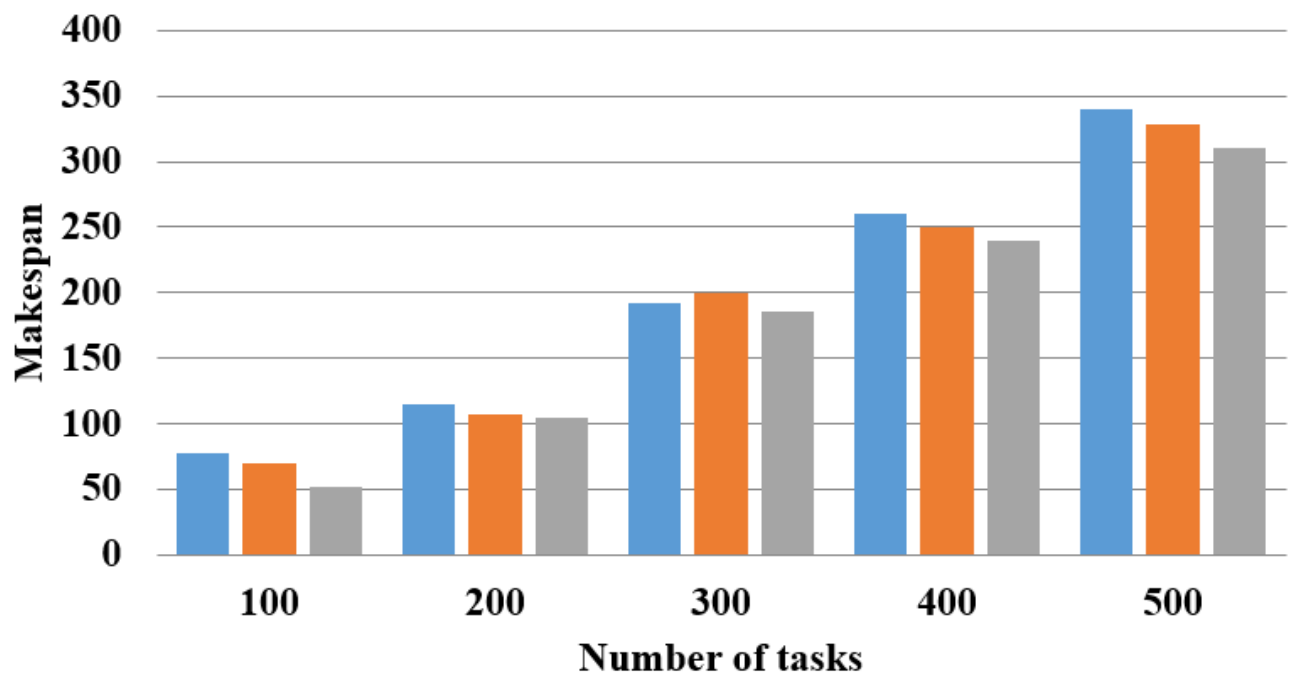

- PSO $\backsim$ Standard GWO Modified MGWO

Figure.3 Makespan for right skewed dataset

Table 2. Energy consumption of proposed modified MGWO

\begin{tabular}{|c|c|c|c|c|c|c|}
\hline \multirow{2}{*}{$\begin{array}{l}\text { Number of } \\
\text { iterations }\end{array}$} & \multicolumn{2}{|c|}{ PSO $[20]$} & \multicolumn{2}{|c|}{ Standard GWO [22] } & \multicolumn{2}{|c|}{ Modified MGWO } \\
\hline & Left skewed & Right skewed & Left skewed & Right skewed & Left skewed & $\begin{array}{l}\text { Right } \\
\text { skewed }\end{array}$ \\
\hline 5 & 25 & 40 & 29 & 49 & 23 & 39 \\
\hline 10 & 22 & 38 & 24 & 40 & 20 & 36 \\
\hline 15 & 20 & 35 & 22 & 32 & 19 & 30 \\
\hline 20 & 17 & 29 & 18 & 28 & 15 & 26 \\
\hline 25 & 18 & 27 & 17 & 25 & 13 & 23 \\
\hline 30 & 16 & 25 & 15 & 20 & 10 & 18 \\
\hline
\end{tabular}




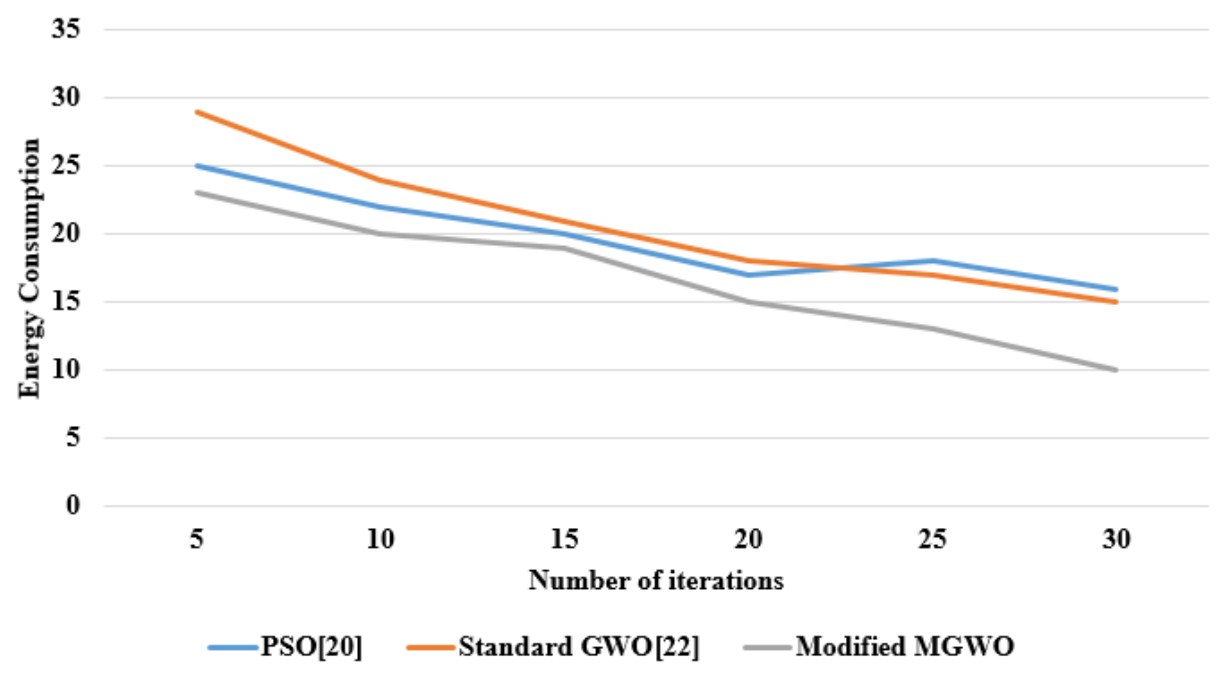

Figure.4 Energy consumption for 400 tasks

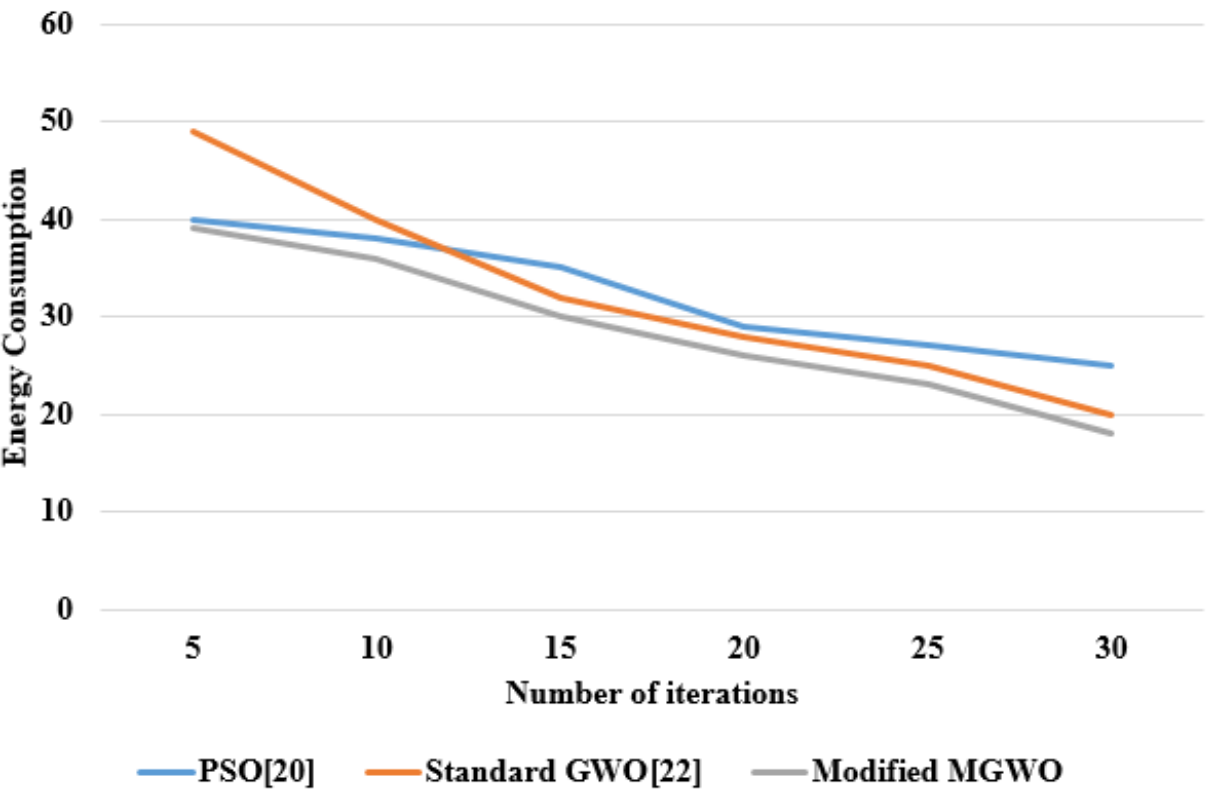

Figure.5 Energy consumption for 400 tasks

From the above Fig. 4, the consumption of energy evaluated by 30 iterations and much reduced by the Modified-MGWO technique, when compared with other existing techniques. In the $5^{\text {th }}$ iterations, the standard GWO consumed high energy because of its fitness function. The PSO achieved $22 \%$ of energy, standard GWO achieved 24\% of energy and MGWO achieved $20 \%$ energy for $10^{\text {th }}$ iterations in LS dataset. When the iterations increase, the consumption of energy reduced for all methods. For instance, at $25^{\text {th }}$ iterations, the PSO achieved 18\% energy, standard GWO achieved $17 \%$ energy and MGWO achieved $13 \%$ energy for LS dataset. The proposed ModifiedMGWO achieved nearly less than 20\% energy consumption for $15^{\text {th }}$ iteration, whereas the GWO achieved $22 \%$ energy consumption.
When the iterations increased, the proposed Modified-MGWO method produced a better result than PSO and standard GWO. Fig. 5 shows the energy consumption of LS data for 400 tasks execution with various iterations. Similarly, the RS data energy consumption is shown in Fig. 4 for 400 tasks with various iterations. For RS dataset, at $5^{\text {th }}$ iterations, all the methods consumed high energy such as PSO consumed 40\% energy, standard GWO consumed 49\% energy and MGWO consumed 39\% energy. As the iterations increases, the consumption of energy decreases. For instance, PSO consumed $25 \%$ energy, standard GWO consumed $20 \%$ energy and proposed MGWO consumed $18 \%$ energy at $30^{\text {th }}$ iterations for RS dataset. During task execution, the modified-MGWO consumed minimum energy for 
both datasets which is validated by experimental analysis for 500 tasks.

\section{Conclusion}

In CC technology, the tasks are mapped with suitable and appropriate resources by TS to execute with various parameters such as resource utilization, execution time, load balancing, cost and so on. The major contribution of this research work is to minimize the consumption of energy and execution time by implementing the Modified MGWO technique on two datasets (LS-RS). The experiments conducted in the CloudSim simulator for validating the performance of Modified MGWO with existing techniques such as PSO and GWO. The MGWO method reduced nearly 13\% MS for 30 iterations and less than $22 \%$ energy consumption for $15^{\text {th }}$ iterations. In future work, the Modified MGWO is integrated with other optimization algorithms to improve the robustness. In addition, more QoS parameters such as the priority of tasks, reliability, load imbalance and security concepts will be considered for better performance.

\section{References}

[1] S.K. Panda and P.K. Jana, "Efficient task scheduling algorithms for heterogeneous multicloud environment", J. Supercomput., Vol.71, No.4, pp.1505-1533, 2015.

[2] Z.H. Zhan, X.F. Liu, Y.J. Gong, J. Zhang, H.S. H. Chung, and Y. Li, "Cloud computing resource scheduling and a survey of its evolutionary approaches", ACM Comput. Surv., Vol.47, No.4, pp.1-33, 2015.

[3] X. Zuo, G. Zhang, and W. Tan, "Self-adaptive learning PSO-based deadline constrained task scheduling for hybrid IaaS cloud", IEEE Trans. Autom. Sci. Eng., Vol.11, No.2, pp.564-573, 2014.

[4] R. Duan, R. Prodan, and X. Li, "Multi-objective game theoretic scheduling of bag-of-tasks workflows on hybrid clouds", IEEE Trans. Cloud Comput., Vol.2, No.1, pp.29-49, 2014

[5] X. Zhu, L.T. Yang, H. Chen, J. Wang, S. Yin, and $\mathrm{X}$. Liu, "Realtime tasks oriented energyaware scheduling in virtualized clouds", IEEE Trans. Cloud Comput., Vol.2, No.2, pp.168-180, 2014.

[6] M.A. Rodriguez and R. Buyya, "Deadline based resqce provisioning and scheduling algorithm for scientific workflows on clouds", IEEE Trans. Cloud Comput., Vol.2, No.2, pp.222-235, 2014.

[7] H. Yuan, J. Bi, W. Tian, M.C. Zhou, B.H. Li, and J. Li, "TTSA: An effective scheduling approach for delay bounded tasks in hybrid clouds", IEEE transactions on cybernetics, Vol.47, No.11, pp.3658-3668, 2017.

[8] N. Jain, I. Menache, J. Naor, and J. Yaniv, "Near-optimal scheduling mechanisms for deadline-sensitive jobs in large computing clusters", ACM Trans. Parallel Comput., Vol.2, No.1, pp.1-29, 2015

[9] C. Delimitrou and C. Kozyrakis, "QoS-aware scheduling in heterogeneous datacenters with paragon," ACM Trans. Comput. Syst., Vol.31, No.4, pp.1-34, 2013.

[10] J. Li, M. Qiu, Z. Ming, G. Quan, X. Qin, and Z. $\mathrm{Gu}$, "Online optimization for scheduling preemptable tasks on IaaS cloud systems", $J$. Parallel Distrib. Comput., Vol.72, No.5, pp.666-677, 2012.

[11] S. Devipriya and C. Ramesh, "Improved Maxmin heuristic model for task scheduling in cloud", In Proc. ICGCE, pp. 883-888, 2013.

[12] J. Zhou and S. Dong, "Hybrid glowworm swarm optimization for task scheduling in the cloud environment", Engine. Optimi, Vol.50, No.6, pp.949-964, 2018.

[13] N. Gobalakrishnan and C. Arun, "Opposition Learning-Based Grey Wolf Optimizer Algorithm for Parallel Machine Scheduling in Cloud Environment", International Journal of Intelligent Engineering and Systems, Vol.10, No.1, pp.186-195.2017.

[14] L. Teylo, U. De Paula, Y. Frota, D. De Oliveira, and L. M. Drummond, "A hybrid evolutionary algorithm for task scheduling and data assignment of dataintensive scientific workflows on clouds", Future Gen. Comput. Syst, Vol.76, pp.1-17, 2017.

[15] C. Delimitrou, D. Sanchez, and C. Kozyrakis, "Tarcil: Reconciling scheduling speed and quality in large shared clusters," In Proc. of International conference on SoCC, pp.97-110, 2015.

[16] J. Li, J. Zhang, C. Jiang, and M. C. Zhou, "Composite particle swarm optimizer with historical memory for function optimization", IEEE Trans. Cybern., Vol.45, No.10, pp.23502363, 2015.

[17] W. Han, J. Xu, M. C. Zhou, G. Tian, P. Wang, X. Shen, and S.-H. E. Hou, "Cuckoo-search and particle-filter-based inversing approach to estimating defects via magnetic flux leakage signals", IEEE Trans. Magn., Vol.52, No.4, pp.1-1, 2016.

[18] H. Yuan, J. Bi, W. Tan, M. Zhou, B. H. Li, and J. Li, "TTSA: An effective scheduling approach for delay bounded tasks in hybrid clouds", IEEE 
Transactions on Cybernetics, Vol.47, No.11, pp.3658-3668, 2017.

[19] H. B. Alla, S. B. Alla, A. Touhafi, and A. Ezzati, "A novel task scheduling approach based on dynamic queues and hybrid meta-heuristic algorithms for cloud computing environment", Cluster Computing, Vol.21, No.4, pp.1797-1820, 2018.

[20] J.A.J. Sujana, T. Revathi, T.S. Priya, and K. Muneeswaran, "Smart PSO-based secured scheduling approaches for scientific workflows in cloud computing", Soft Computing, Vol.23, No.5, pp.1745-1765, 2017.

[21] C. Thirumalaiselvan and V. Venkatachalam, "A strategic performance of virtual task scheduling in multi cloud environment", Cluster Computing, pp.1-9, 2017.

[22] G. Natesan and A. Chokkalingam, "Opposition Learning-Based Grey Wolf Optimizer Algorithm for Parallel Machine Scheduling in Cloud Environment", International Journal of Intelligent Engineering and Systems, Vol.10, pp.186-195, 2017.

[23] T. Latha, "QoS based dynamic task scheduling in IaaS cloud", In: Proc. of International Conference on Recent Trends in Information Technology, pp.1-8, 2014.

[24] S. Mirjalili, S. M. Mirjalili, and A. Lewis, "Grey wolf optimizer", Advances in Engineering Software, Vol.69, pp.46-61, 2014. 